\title{
REFINED ALGEBRAIC QUANTIZATION: SYSTEMS WITH A SINGLE CONSTRAINT
}

\author{
DONALD MAROLF \\ Physics Department, University of California \\ Santa Barbara, California 93106, USA \\ E-mail: marolf@cosmic.physics.ucsb.edu
}

\begin{abstract}
This paper explores in some detail a recent proposal (the Rieffel induction/refined algebraic quantization scheme) for the quantization of constrained gauge systems. Below, the focus is on systems with a single constraint and, in this context, on the uniqueness of the construction. While in general the results depend heavily on the choices made for certain auxiliary structures, an additional physical argument leads to a unique result for typical cases. We also discuss the 'superselection laws' that result from this scheme and how their existence also depends on the choice of auxiliary structures. Again, when these structures are chosen in a physically motivated way, the resulting superselection laws are physically reasonable.
\end{abstract}

1. Introduction. Canonical quantization of gauge systems has been a subject of much discussion since the basic outline was first given by Dirac [5]. This formalism has been especially popular in the gravitational physics community as, for Einstein's general theory of relativity on a spatially compact universe, the Hamiltonian consists only of constraints. In addition, the nature of the gauge transformations associated with gravity make gauge fixing techniques extremely difficult to apply and perturbative nonrenormalizability has frustrated attempts at covariant path integral quantization. Thus, Dirac style canonical quantization remains at the forefront of quantum gravity research $[1,10,11]$.

Despite this interest, certain basic issues have remained unresolved for the general case. Recall that the essential idea of Dirac's approach is to turn the classical constraints $C_{i}$ into linear operators $\widehat{C}_{i}$ and to consider 'physical states $\psi_{\text {phys' }}$ ' that are annihilated by the constraints; i.e., such that $\widehat{C}_{i} \psi_{\text {phys }}=0$. However, the questions of on which linear space the constraints should act and of just how an inner product is to be imposed on the solutions to define a Hilbert space do not yet have widely accepted answers.

Recently, a resolution to these issues has been proposed. In fact, what is essentially the

1991 Mathematics Subject Classification: Primary 81V17; Secondary 81Q10.

This work was supported by NSF grant PHY90-08502.

The paper is in final form and no version of it will be published elsewhere. 
same resolution has been independently suggested twice under the names of the 'Rieffel induction procedure' [12] and the 'refined algebraic quantization scheme' [2]. This method has been successfully used to quantize linearized gravity on symmetric backgrounds [8,9], minisuperspace models for gravity [17,18], and the free Maxwell field [14]. As might be expected, these methods proceed by introducing additional structures beyond what is present in the original Dirac approach. These techniques and additional structures will be explored further here in the particular context of systems with a single constraint.

We begin with a review of the Rieffel/refined algebraic procedure in Section 2. Here, we use the language and notation of [2] as it is more closely related to that of Dirac [5] and therefore more familiar. We will also refer to the scheme as the 'refined algebraic proposal' in the text.

Sections 3 and 4 contain the main results of this paper. In Section 3 we show how, for typical systems with a single constraint, a physical argument determines a unique implementation of the Rieffel/refined algebraic scheme. In Section 4 we discuss superselection laws on the physical Hilbert space and how their existence may depend on the choice of auxiliary structures. We give two examples. In the first, the use of an 'incorrect' structure leads to spurious superselection laws. This example also illustrates the fact that the physical Hilbert space can depend strongly on the choice of this structure. In the second, the use of a physically motivated auxiliary structure produces superselection laws, but this time a similar feature exists in the classical theory. Thus, for this second case we take the superselection laws to be physically meaningful. Appendices A and B contain proofs of technical results which are not of direct relevance to the main discussion but which are mentioned in the text.

2. The Refined Algebraic Approach. In this section, we review the refined algebraic quantization scheme presented in [2] (which is essentially equivalent to the Rieffel induction procedure of [12]) for systems with gauge symmetries. The starting point is a constrained classical system with phase space $\Gamma$ and, as usual, the nondegenerate symplectic form $\omega$ on $\Gamma$ defines a Poisson bracket on smooth functions $\Gamma \rightarrow \mathbb{C}$. The constraints $C_{i}$ are required to be first class; that is, the Poisson bracket of two constraints is a sum of constraints (possibly weighted by smooth phase space functions), as is the Poisson bracket of any constraint with the Hamiltonian. As a result, the constraint surface is preserved under time evolution.

The refined algebraic proposal quantizes this system in a series of steps based on those of the original algebraic quantization program $[1,3]$. The first four steps below have nothing to do with constrained systems but simply quantize the system obtained by ignoring the constraints. They follow the unconstrained prescription of $[1,3]$ exactly but we repeat them here for completeness and to fix our notation.

Step 1. Select a subspace $\mathbf{S}$ of the vector space of all smooth, complex-valued functions on $\Gamma$ subject to the following conditions:

a) $\mathbf{S}$ should be large enough so that any sufficiently regular function on the phase space can be obtained as (possibly a suitable limit of) a sum of products of elements in $\mathbf{S}$.

b) $\mathbf{S}$ should be closed under Poisson brackets, i.e. for all functions $F, G$ in $\mathbf{S}$, their 
Poisson bracket $\{F, G\}$ should also be an element of $\mathbf{S}$.

c) Finally, $\mathbf{S}$ should be closed under complex conjugation; i.e. for all $F$ in $\mathbf{S}$, the complex conjugate $F^{*}$ should be a function in $\mathbf{S}$.

The idea is that each function in $\mathbf{S}$ is to be regarded as an elementary classical variable which is to have an unambiguous quantum analogue.

Step 2. Associate with each element $F$ in $\mathbf{S}$ an abstract operator $\widehat{F}$. Construct the free associative algebra generated by these elementary quantum operators. Impose on it the canonical commutation relations, $[\widehat{F}, \widehat{G}]=i \hbar \widehat{\{F, G\}}$, and, if necessary, also a set of (anti-commutation) relations that captures the algebraic identities satisfied by the elementary classical variables. Denote the resulting algebra by $\mathcal{B}_{\text {aux }}$.

Step 3. On this algebra, introduce an involution $\left({ }^{1}\right)$ operation $\star$ by requiring that if two elementary classical variables $F$ and $G$ are related by $F^{*}=G$, then $\widehat{F}^{\star}=\widehat{G}$ in $\mathcal{B}_{\text {aux }}$. Denote the resulting $\star$-algebra by $\mathcal{B}_{\text {aux }}^{(\star)}$.

Step 4. Construct a linear $\star$-representation $R$ of the abstract algebra $\mathcal{B}_{\text {aux }}^{(\star)}$ via linear operators on an auxiliary Hilbert space $\mathcal{H}_{\text {aux }}$, i.e. such that

$$
R\left(\widehat{A}^{\star}\right)=R(\widehat{A})^{\dagger}
$$

for all $\widehat{A}$ in $\mathcal{B}^{(\star)}$, where $\dagger$ denotes Hermitian conjugation with respect to the inner product in $\mathcal{H}_{\text {aux }}$.

The remaining steps introduce the constraints and address the questions raised in the introduction. That is, they first use the space $\mathcal{H}_{\text {aux }}$ to provide a home for the constraints and for the linear space on which they act, and then construct the physical Hilbert space from the corresponding solutions.

Step 5a. Represent the constraints $C_{i}$ as self-adjoint operators $\widehat{C}_{i}$ (or, their exponentiated action, representing the finite gauge transformations, as unitary operators $\widehat{U}_{i}$ ) on $\mathcal{H}_{\text {aux }}$.

We will now look for solutions of the constraints in terms of generalized eigenvectors of $\widehat{C}_{i}$ which will lie in the topological dual $\Phi^{\prime}$ of some dense subspace $\Phi \subset \mathcal{H}_{\text {aux }}$ (see also $[6,7])$. Since $\Phi$ and $\Phi^{\prime}$ will be used to build the physical Hilbert space, we will consider only physical operators that are well behaved with respect to $\Phi$.

Step 5b. Choose a suitable dense subspace $\Phi \subset \mathcal{H}_{\text {aux }}$ which is left invariant by the constraints $\widehat{C_{i}}$ and let $\mathcal{B}_{\text {phys }}^{(\star)}$ be the $\star$-algebra of operators on $\mathcal{H}_{\text {aux }}$ which commute with the constraints $\widehat{C}_{i}$ and such that, for $A \in \mathcal{B}_{\text {phys }}^{(\star)}$, both $A$ and $A^{\dagger}$ are defined on $\Phi$ and map $\Phi$ to itself.

As an example in Section 4 will illustrate, some physical input is in general required to choose the space $\Phi$. Some factors governing this choice are that it must be sufficiently

$\left.{ }^{1}\right)$ Recall that an involution on $\mathcal{B}_{\text {aux }}$ is an anti-linear map $\star$ from $\mathcal{B}_{\text {aux }}$ to itself satisfying the following three conditions for all $A$ and $B$ in $\mathcal{B}_{\text {aux }}$ : i) $(A+\lambda B)^{\star}=A^{\star}+\lambda^{*} B^{\star}$, where $\lambda$ is any complex number; ii) $(A B)^{\star}=B^{\star} A^{\star}$; and iii) $\left(A^{\star}\right)^{\star}=A$. 
large so that $\mathcal{B}_{\text {phys }}^{(\star)}$ contains 'enough' physically interesting operators while it must also be sufficiently small that its topological dual $\Phi^{\prime}$ contains enough physical states.

The main idea in the last few steps is that, while the classical reality conditions should determine the inner product, we should not need to explicitly display a complete set of classical observables (i.e., functions which Poisson commute with the constraints) to achieve this goal. Instead, we use a complete set of functions $(\mathbf{S})$ on the unconstrained phase space, noting that the reality properties of such functions will determine the reality properties of the observables. The reality conditions of operators in $\mathcal{B}_{\text {aux }}^{(\star)}$ are then implemented on the auxiliary Hilbert space $\mathcal{H}_{\text {aux }}$. The physical Hilbert space $\mathcal{H}_{\text {phys }}$ is to be constructed in such a way that any adjointness relations involving only observables (i.e., $A=B^{\dagger}$, for $A, B$ observables) will in turn descend from $\mathcal{H}_{\text {aux }}$ to $\mathcal{H}_{\text {phys }}$ (so that $A=B^{\dagger}$ on $\mathcal{H}_{\text {phys }}$ as well). In this way, we will say that the reality conditions are implemented on $\mathcal{H}_{\text {phys }}$.

We now wish to construct the physical Hilbert space $\mathcal{H}_{\text {phys }}$, which will in general not be a subspace of $\mathcal{H}_{\text {aux }}$. The key idea is to find an appropriate map $\eta: \Phi \rightarrow \Phi^{\prime}$ such that $\eta(\phi)$ is a solution of the constraints for all $\phi \in \Phi$. (Note that the natural class of maps from $\Phi$ to $\Phi^{\prime}$ is anti-linear (e.g., the adjoint map)). We proceed as follows.

Step 5c. Find an anti-linear map $\eta$ from $\Phi$ to the topological dual $\Phi^{\prime}$ that satisfies:

(i) For every $\phi_{1} \in \Phi, \eta\left(\phi_{1}\right)$ is a solution of the constraints; i.e.,

$$
0=\left(\widehat{C}_{i}\left(\eta \phi_{1}\right)\right)\left[\phi_{2}\right]:=\left(\eta \phi_{1}\right)\left[\widehat{C}_{i} \phi_{2}\right]
$$

for any $\phi_{2} \in \Phi$. Here, the square brackets denote the natural action of $\Phi^{\prime}$ on $\Phi$.

(ii) $\eta$ is real and positive in the sense that, for all $\phi_{1}, \phi_{2} \in \Phi$,

$$
\left(\eta \phi_{1}\right)\left[\phi_{2}\right]=\left(\left(\eta \phi_{2}\right)\left[\phi_{1}\right]\right)^{*} \quad \text { and } \quad\left(\eta \phi_{1}\right)\left[\phi_{1}\right] \geq 0 .
$$

(iii) $\eta$ commutes with the action of any $A \in \mathcal{B}_{\text {phys }}^{(\star)}$ in the sense that

$$
\left(\eta \phi_{1}\right)\left[A \phi_{2}\right]=\left(\left(\eta A^{\dagger} \phi_{1}\right)\right)\left[\phi_{2}\right]
$$

for all $\phi_{1}, \phi_{2} \in \Phi$. The right-hand side defines the so-called dual action of $A$ on $\Phi^{\prime}$ so that we may write this as $\eta A \phi=A \eta \phi$.

In analogy with [12] we call $\eta$ the rigging map. (The appearance of the adjoint on the right-hand side of the above equation corresponds to the anti-linearity of $\eta$.)

Step 5d. The vectors $\eta \phi$ span a space $\mathcal{V}_{\text {phys }}$ of solutions of the constraints. We introduce an inner product on $\mathcal{V}_{\text {phys }}$ through

$$
\left\langle\eta \phi_{1}, \eta \phi_{2}\right\rangle_{\mathrm{phys}}=\left(\eta \phi_{2}\right)\left[\phi_{1}\right]
$$

The requirement (iii) guarantees that this inner product is well defined and that it is Hermitian and positive definite so that the corresponding completion of $\mathcal{V}_{\text {phys }}$ is a 'physical' Hilbert space $\mathcal{H}_{\text {phys }}$. (Note that the positions of $\phi_{1}$ and $\phi_{2}$ must be opposite on the two sides of this definition due to the anti-linear nature of $\eta$.)

At this point, the reader may fear that this list of conditions on $\eta$ will never be met in practice. That the new Step 5 may actually simplify the quantization program follows 
from the observation of $[8,9]$ (and $[15,16,17]$ for the case when the Poisson algebra of constraints is Abelian) that natural candidates for such a map exist.

The last step is to represent physical operators on $\mathcal{V}_{\text {phys }}$. This is straightforward because the framework provided by Step 5 guarantees that $\mathcal{H}_{\text {phys }}$ carries an (anti) $\star$-representation (see below) of $\mathcal{B}_{\text {phys }}^{(\star)}$ as follows:

Step 6. Operators in $A \in \mathcal{B}_{\text {phys }}^{(\star)}$ have a natural action (induced by duality) on $\Phi^{\prime}$ that leaves $\mathcal{V}_{\text {phys }}$ invariant. Use this fact to induce densely defined operators $A_{\text {phys }}$ on $\mathcal{H}_{\text {phys }}$ through

$$
A_{\text {phys }}(\eta \phi)=\eta(A \phi) \text {. }
$$

This leads to an anti- $\star$-representation of $\mathcal{B}_{\text {phys }}^{(\star)}$ on $\mathcal{H}_{\text {phys }}$ as the map $A \mapsto A_{\text {phys }}$ from $\mathcal{B}_{\text {phys }}^{(\star)}$ to the operators on $\mathcal{H}_{\text {phys }}$ is an anti-linear $\star$-homomorphism where $\star$ acts on the operator $A_{\text {phys }}$ in the sense of conjugation of quadratic forms on the dense domain $\Phi$ $\left(\left\langle\phi, A^{\star} \psi\right\rangle \equiv\langle\psi, A \phi\rangle^{*}\right)$. In this way, the reality properties of the physical operators $\mathcal{B}_{\text {phys }}^{(\star)}$ on $\mathcal{H}_{\text {aux }}$ descend to the physical Hilbert space.

In addition, note that the collection of all bounded operators in $\mathcal{B}_{\text {phys }}^{(\star)}$ in fact defines a $C *$-algebra $\mathcal{B}^{C *}$ with unit. Since the physical expectation value $\eta(A \phi)[\phi]$ defines a positive functional on $\mathcal{B}^{C *}$ (i.e., $\eta\left(A^{\dagger} A \phi\right)[\phi] \geq 0$ ), it follows that for $A \in \mathcal{B}^{C *}$ we have

$$
\eta\left(A^{\dagger} A \phi\right)[\phi] \leq\|A\|^{2} \eta(\phi)[\phi]
$$

so that $A_{\text {phys }}$ is a bounded operator on $\mathcal{H}_{\text {phys }}$ with norm not larger than that of $A$ on $\mathcal{H}_{\text {aux }}\left(\|A\|_{\text {phys }} \leq\|A\|\right)$. Thus, for such bounded operators, any relations of the form $A=B^{\dagger}$ on $\mathcal{H}_{\text {aux }}$ also holds as the adjointness relation $A_{\text {phys }}=B_{\text {phys }}^{\dagger}$ on $\mathcal{H}_{\text {phys }}$. From this it follows that if $A$ is self-adjoint on $\mathcal{H}_{\text {aux }}$, then $\mathcal{B}_{\text {phys }}^{(\star)}$ determines a (unique) self-adjoint extension of $A_{\text {phys }}$ on $\mathcal{H}_{\text {phys }}$.

Let us consider for a moment the case where there is only one constraint. Note that when this constraint has purely discrete spectrum, there is a natural choice for the map $\eta$ as follows. Let $\Pi_{0}$ be the projection onto the eigenspace of the constraint with eigenvalue zero. Then if we take $\Phi=\mathcal{H}_{\text {aux }}$, the rigging map $\eta$ given by

$$
\eta|\psi\rangle=\langle\psi| \Pi_{0}
$$

fulfills all the requirements of Step 5c. This case is simple and easy to deal with, so that we shall focus on the complimentary case (where the spectrum is purely continuous) in the next section. Section 4 will describe what happens when both continuous and discrete spectra are present.

3. A Unique Prescription. While the framework described in Section 2 sets the stage for quantizing constrained systems, it does not provide the complete script. There are in fact three inputs that need to be provided in order to proceed. The first is the auxiliary space $\mathcal{H}_{\text {aux }}$ itself, but the dense subspace $\Phi \subset \mathcal{H}_{\text {aux }}$ and the rigging map $\eta$ : $\Phi \rightarrow \Phi^{\prime}$ must also be given. As such, it is natural to ask to what extent the above prescription is unique and to what extent it depends on the choice of these inputs. In general, the answer is that the final formulation depends a great deal on the inputs, as 
different choices can even lead to physical Hilbert spaces of different dimensions! This will be illustrated by an example in the next section.

Below, we confine ourselves to the case of a single constraint $\widehat{C}$ of the typical kind that arises in finite dimensional models. The two main types of constrained systems are the 'classic' gauge systems in which the constraint is a vector field (whose orbits are closed subsets of the configuration space) on some configuration space and the 'time reparametrization invariant systems' in which the constraint is essentially the same as some Hamiltonian of nonrelativistic quantum mechanics (but typically with both positive and negative kinetic terms). For such cases, physical reasoning will lead to a preferred choice of the dense subspace $\Phi$ such that the rigging map is then unique up to scale. As we consider constraints with continuous spectrum, we shall assume that the configuration space is noncompact. We argue as follows.

An important element of classical symplectic mechanics is that the algebra of observables is taken to be the set of smooth functions on the phase space (as in Step 1 of the refined algebraic program). It is this definition, for example, that allows us to talk about the (local) symplectomorphism 'generated by an observable $A$ '. As such, the topology and differential structure of the phase space play a key role and we would like to encode them in our quantum formulation. Consider the case where the classical phase space is $T^{*} \mathbb{R}^{n}$ and the auxiliary Hilbert space used in the refined quantization program is $L^{2}\left(\mathbb{R}^{n}\right)$. Recall that one characterization of the Schwarz space $\mathcal{S} \subset \mathcal{H}_{\text {aux }}$ is as the set of all states $|\psi\rangle$ for which both $\langle x \mid \psi\rangle$ and $\langle p \mid \psi\rangle$ are smooth $L^{2}$ functions of $x$ and $p$, where $\langle x|$ and $\langle p|$ are the usual position and momentum generalized eigenstates. Thus, this set of states can be said to encode the differential structure of the classical phase space and is a natural choice for the subspace $\Phi$ of Step $5 \mathrm{~b}$. The algebra $\mathcal{B}_{\text {phys }}^{(\star)}$ of operators that preserve this space contains all suitably smooth and rapidly decreasing combinations of $x$ and $p$, in good analogy with the classical algebra of observables. Thus, we take $\Phi=\mathcal{S}$.

We will now show how a rigging map $\eta$ can be defined using this choice and that this map is unique (given $\Phi=\mathcal{S}$ ). Unfortunately, rigorous results are known to the author only when certain additional assumptions are placed on the constraints (which will be described below), but it is reasonable to conjecture that similar results hold in the general case.

The result we need for our system is the following:

Property A. There exists a set of generalized states $\langle c, k|$ for $c \in D_{C}, D_{C}$ an open subset of $\mathbb{R}$ containing 0 , and $k \in D_{K}, D_{K}$ an open subset of $\mathbb{R}^{n-1}$, satisfying $\langle c, k| \widehat{C}=\langle c, k| c$ and $\left\langle c, k \mid c^{\prime}, k^{\prime}\right\rangle=\delta\left(c-c^{\prime}\right) \delta\left(k-k^{\prime}\right)$ and which are complete on the closed subspace of $\mathcal{H}_{\text {aux }}$ corresponding to the open spectral interval $D_{C}$ of $\widehat{C}$. The $\langle c, k|$ are elements of the (algebraic) dual $\mathcal{S}^{\text {dual }}$ to $\mathcal{S}$ and the map $F_{k}: c \mapsto\langle c, k|$ is continuous with respect to the pointwise convergence topology on $\mathcal{S}^{\text {dual }}$. Furthermore, the map $F$ : $D_{C} \times \mathcal{S} \rightarrow L^{2}\left(D_{K}, d^{n-1} k\right)$ given by $F:(c,|\psi\rangle) \mapsto|\psi\rangle_{c}$ such that $\langle k \mid \psi\rangle_{c}=\langle c, k \mid \psi\rangle$ is well-defined and continuous.

Such a result is easy to derive when the constraint is a vector field with sufficiently regular orbits by simply introducing coordinates in the space of orbits. For the case of a Hamiltonian constraint, we will need to say something more about the form of the 
Hamiltonian. Results are known for the following special cases:

1. The massive free particle: Property A may be checked directly using the momentum eigenstates.

2. The so-called separable semi-bound cases (see [17]): It follows from the integral representation 5.14 of [17] that, when a scattering operator exists for the 'transverse' Hamiltonian $H_{1}$, there is a complete set of orthogonal and appropriately normalized generalized eigenstates $\langle c, k|$ satisfying Property $\mathrm{A}$.

3. When the constraint is of the form $H=\sum_{i} p_{i}^{2}+V(q)-E$ and $V \in L^{1}$ : By extending Lemma IV.28 of $[20]$ from $C_{0}^{\infty}\left(\mathbb{R}^{n}\right)$ to $\mathcal{S}$, Property A reduces to the requirement that $H$ have purely continuous spectrum.

Unfortunately, the literature contains less helpful results than one would like. This is largely due to the fact that Hamiltonian constraints tend not to have positive definite kinetic terms, while the literature is primarily concerned with the Hamiltonians of particles moving on a Riemannian space. Nevertheless, case 2 above contains nontrivial cosmological models and we suspect that Property A in fact holds in more general situations. We will therefore assume that our system has Property A without further justification.

Now, for $|\phi\rangle \in \mathcal{S}$, let $\phi(c, k)$ be the function $\langle c, k \mid \psi\rangle$. Using Property A, we can construct the rigging map $\eta_{0}$ through

$$
\left(\eta_{0} \phi_{1}\right)\left[\phi_{2}\right]=\int d c \delta(c) \int d k \phi_{1}^{*}(c, k) \phi_{2}(c, k)
$$

which clearly satisfies the criteria of Step 5c. Note that the action of the delta function is well defined since $\phi_{1}$ and $\phi_{2}$ are continuous in $c$ by Property A.

We will now see that this is the unique map (up to an overall scale) that satisfies $5 \mathrm{c}$. To do so, consider some generic rigging map $\eta$. Since $\eta$ must commute with the constraint, but has only solutions of the constraint in its image, it is clear that $\eta$ must annihilate the domain of $\widehat{C}^{-1}$; that is, all $|\phi\rangle \in \mathcal{S}$ for which $\phi(0, k)=0$. It follows that the kernel of $\eta$ includes the kernel of $\eta_{0}$.

Let us now consider two states $\left|\phi_{1}\right\rangle,\left|\phi_{2}\right\rangle \in \mathcal{S}$ which are not annihilated by $\eta_{0}$; that is, for which $\phi_{1}(0, k)$ and $\phi_{2}(0, k)$ are nonzero on a positive measure subset of $D_{K}$. Then by continuity there is some $\epsilon$ such that

$$
\int d k\left|\phi_{i}(c, k)\right|^{2}>0 \quad(i=1,2)
$$

for all $|c|<\epsilon$ and such that $[-\epsilon, \epsilon] \subset D_{C}$. We now define $\Pi_{[-\epsilon, \epsilon]}$ to be the projection onto the spectral interval $[-\epsilon, \epsilon]$ of the constraint $\widehat{C}$ and consider the state

$$
\left|\psi_{1}\right\rangle=\Pi_{[-\epsilon, \epsilon]}\left|\phi_{1}\right\rangle \text {. }
$$

Note that $\left|\psi_{1}\right\rangle$ and $\left|\phi_{1}\right\rangle$ map to the same element of $\Phi^{\prime}$ under both $\eta$ and $\eta_{0}$. We also define a state $\left|\psi_{2}\right\rangle$ by the equation

$$
\psi_{2}(c, k)=\sqrt{\frac{\int d k^{\prime}\left|\phi_{1}\left(c, k^{\prime}\right)\right|^{2}}{\int d k^{\prime}\left|\phi_{2}\left(c, k^{\prime}\right)\right|^{2}}} \phi_{2}(c, k)
$$

for $|c| \leq \epsilon$ and $\phi_{2}(c, k)=0$ for $|c|>\epsilon$. While $\left|\phi_{2}\right\rangle$ and $\left|\psi_{2}\right\rangle$ map to different elements of $\Phi^{\prime}$, they map to the same ray in $\mathcal{H}_{\text {phys }}$ under $\eta$ and to the same ray in $\mathcal{H}_{\text {phys }, 0}$ under $\eta_{0}$. 
Note that $\eta_{0}\left|\psi_{1}\right\rangle$ and $\eta_{0}\left|\psi_{2}\right\rangle$ have the same norm in $\mathcal{H}_{\text {phys }, 0}$, but are otherwise arbitrary elements of $\mathcal{H}_{\text {phys }, 0}$.

We will now show that the conditions of Step 5c guarantee that $\eta\left|\psi_{1}\right\rangle$ and $\eta\left|\psi_{2}\right\rangle$ have the same physical norm no matter how $\eta$ is defined. To proceed, consider the family $U(\theta)$ of unitary operators that generate rotations in the two dimensional subspace of $\mathcal{H}_{\text {aux }}$ spanned by $\left|\psi_{1}\right\rangle$ and $\left|\psi_{2}\right\rangle$ and note that, for fixed $c$, the functions $\psi_{i}(c, k)$ define elements $\left|\psi_{i, c}\right\rangle$ of the 'transverse' Hilbert space $\mathcal{H}_{c} \sim L^{2}\left(D_{K}, d k\right)$. Such $U(\theta)$ are in fact diagonal in $c$; that is, they satisfy

$$
\langle c, k|U(\theta)| \phi\rangle=\left\langle k\left|U_{c}(\theta)\right| \phi_{c}\right\rangle_{c}
$$

where the subscripts $c$ on the right-hand side indicate that the matrix element is taken in the transverse Hilbert space $\mathcal{H}_{c}$. Here, $U_{c}(\theta)$ is just the unitary operator on $\mathcal{H}_{c}$ that rotates the subspace spanned by $\left|\psi_{1, c}\right\rangle$ and $\left|\psi_{2, c}\right\rangle$ and $\langle k|$ is the ket for which $\left\langle k \mid \phi_{c}\right\rangle_{c}=$ $\phi(c, k)$. As a result, $U(\theta)$ commutes with the constraint $\widehat{C}$ and, since it preserves the subspace $\Phi$, must belong to the algebra $\mathcal{B}_{\text {phys }}^{(\star)}$ of observables. However, this means that it must commute with $\eta$ and define a unitary operator on the corresponding physical Hilbert space. It follows that whenever $\left(\eta_{0} \psi_{1}\right)\left[\psi_{1}\right]=\left(\eta_{0} \psi_{2}\right)\left[\psi_{2}\right]$, we must also have $\left(\eta \psi_{1}\right)\left[\psi_{1}\right]=$ $\left(\eta \psi_{2}\right)\left[\psi_{2}\right]$. Since $\eta$ provides a positive semidefinite inner product, the functional $\phi \mapsto$ $(\eta \phi)[\phi]$ in fact defines $\eta$ completely and $\eta$ must be just $\eta_{0}$ up to some overall positive scale factor.

4. Superselection Laws. In contrast with the previous section, the case considered in [2] did not result in a unique rigging map. Instead, a large family of maps was found, associated with the existence of certain 'superselection rules'. It seems a reasonable conjecture that, for a given choice of subspace $\Phi$, the non-uniqueness of the rigging map is always exactly determined by the superselection rules. While we shall not prove this here, the discussion below provides supporting evidence. Appendix A shows that this is true for the particular case studied in [2].

Interestingly, the very existence of superselection rules can depend on the choice of the dense subspace $\Phi$ of Step 5b. This emphasizes the importance of choosing $\Phi$ based on physical motivations. Below, we provide two examples of cases where a superselection laws arises: one (in 4.1) in which it seems to come from the 'wrong' choice of $\Phi$, and one (in 4.2) in which its existence reflects a feature of the classical physics.

4.1. The dependence on $\Phi$. For our first example, we will rework the case of Section 2 using a different choice of $\Phi$. Property A allows us to introduce a notion of continuous states as follows:

Definition. A state $|\phi\rangle \in \mathcal{H}_{\text {aux }}$ is said to be continuous on $\Sigma \subset D_{C}$ if $\phi(c, k)$ is continuous in $c$ for each fixed $k$ at every $c \in \Sigma$.

We will construct $\Phi$ in the following (complicated!) way. Choose some interval $[-a, a] \subset D_{C}$. Now, consider the subintervals $I_{n}^{-}=\left(-\frac{a}{2^{n}},-\frac{a}{2^{n+1}}\right)$ and $I_{n}^{+}=\left(\frac{a}{2^{n+1}}, \frac{a}{2^{n}}\right)$ for $n \geq 0$. Let $R_{E}$ be the union of the $I_{n}^{ \pm}$for even $n$ and $R_{O}$ be the union for odd $n$. In addition, consider a family of projections $\Pi_{c}$ on $\mathcal{H}_{c}$ for which the matrix elements 
$\left\langle k\left|\Pi_{c}\right| k^{\prime}\right\rangle_{c}$ are independent of $c$ and let $\mathcal{N}_{c}$ be the subspace of $\mathcal{H}_{c}$ annihilated by $\Pi_{c}$. We now let $\Phi$ be the dense subspace of $\mathcal{H}_{\text {aux }}$ containing all states $|\psi\rangle$ such that

A) $|\psi\rangle$ is continuous on $R_{E}$ and $\lim _{c \rightarrow 0}$ in $R_{E}$ exists in $\mathbb{C}$.

B) $|\psi\rangle$ is continuous on $R_{O}$ and $\lim _{c \rightarrow 0}$ in $R_{O}$ exists in $\mathbb{C}$.

C) $\Pi_{c}\left|\psi_{c}\right\rangle=0$ at the midpoint of $I_{n}^{ \pm}$for each odd $n$.

The limit in $\mathrm{A}(\mathrm{B})$ is taken by considering only sequences in $R_{E}\left(R_{O}\right)$.

Note that since elements of $\Phi$ are only required to be continuous separately on the sets $R_{E}$ and $R_{O}$, there are now two natural choices for the rigging map, $\eta_{E}$ and $\eta_{O}$ :

$$
\begin{aligned}
& \left(\eta_{E} \phi\right)[\psi]=\lim _{c \rightarrow 0 \text { in } R_{E}} \int d k \phi^{*}(c, k) \psi(c, k) \\
& \left(\eta_{O} \phi\right)[\psi]=\lim _{c \rightarrow 0 \text { in } R_{O}} \int d k \phi^{*}(c, k) \psi(c, k)
\end{aligned}
$$

Remark 1. Note that $\eta_{E}$ leads to the usual physical Hilbert space $L^{2}\left(D_{K}, d k\right)$, whereas $\eta_{O}$ leads to a smaller physical space isomorphic to $\mathcal{N}_{c}$.

Remark 2. For fans of group averaging, we mention that the group averaging procedure $[2,8,9,12]$ does not converge on $\Phi$ (see Appendix B).

The existence of these two maps is associated with the following superselection rule. Let $\Phi_{E} \subset \Phi$ contain those states of $|\psi\rangle$ for which $\psi(c, k)=0$ when $c \in R_{O}$ and let $\Phi_{O} \subset \Phi$ contain those for which $\psi(c, k)=0$ when $c \in R_{E}$. Then, for any $A \in \mathcal{B}_{\text {phys }}^{(\star)}$, because $[A, C]=0$, we have $\left\langle\phi_{E}|A| \phi_{O}\right\rangle=0$ for any $\left|\phi_{E}\right\rangle \in \Phi_{E},\left|\phi_{O}\right\rangle \in \Phi_{O}$. Such superselection rules then descend to the physical level; that is, to the action of the physical operators on the physical Hilbert space.

For a general constraint (such as, say, $p_{x}=0$, generating translation gauge invariance), there is no reason to expect superselection rules. Also, the Hilbert space that results from $\eta_{O}$ seems unreasonably small. Thus, we must regard these features as artifacts of using the 'wrong' choice of $\Phi$. In contrast, the physically motivated choice of Section 3 produced perfectly satisfactory results.

4.2. Physical superselection laws. We now turn an example the superselection rule captures a feature of the corresponding classical system, and thus appears physically meaningful. For this case, we consider systems which differ slightly from those considered so far. We now ask only that our system satisfy 'Property B':

Property B. The Hilbert space $\mathcal{H}_{\text {aux }}$ can be written as a direct sum $\mathcal{H}_{\text {aux }}=\mathcal{H}_{\text {disc }} \oplus$ $\mathcal{H}_{\text {cont }}$ where $\mathcal{H}_{\text {disc }}$ is (densely) spanned by normalizable eigenstates of $\widehat{C}$ and such that when the system is restricted to $\mathcal{H}_{\text {cont }}$, it satisfies Property A.

Now, let $\Phi=\mathcal{H}_{\text {disc }} \oplus \Pi_{\text {cont }} \mathcal{S}$ where $\Pi_{\text {cont }}$ is the projection to $\mathcal{H}_{\text {cont }}$. Again, there are two natural choices of rigging map. First is $\eta_{\text {disc }}$,

$$
\eta_{\text {disc }}|\psi\rangle=\langle\psi| \Pi_{0}
$$

where $\Pi_{0}$ is the projection onto the (normalizable) eigenstates of $\widehat{C}$ with eigenvalue zero. Second is $\eta_{\text {cont }}$, defined to annihilate $\mathcal{H}_{\text {disc }}$ but otherwise just as in Section 3 . Any 
combination $a \eta_{\text {disc }}+b \eta_{\text {cont }}$ for $a, b>0$ also defines a rigging map that satisfies the requirements of Step $5 c$.

Again, there is an associated superselection law between $\mathcal{H}_{\text {disc }}$ and $\Pi_{\text {cont }} \mathcal{S}$. To see this, note that since $A \in \mathcal{B}_{\text {phys }}^{(\star)}$ has an adjoint $A^{\dagger} \in \mathcal{B}_{\text {phys }}^{(\star)}$, we need only show that, for all $A \in \mathcal{B}_{\text {phys }}^{(\star)}, A$ maps $\mathcal{H}_{\text {disc }}$ into $\mathcal{H}_{\text {disc }}$ and we will be done. However, since $[A, \widehat{C}]=0$ and the domain of $A$ contains $\Phi \supset \mathcal{H}_{\text {disc }}, A$ must map every normalizable eigenvector of $\widehat{C}$ to a normalizable eigenvector of $\widehat{C}$ (with the same eigenvalue). Thus, each $A \in \mathcal{B}_{\text {phys }}^{(\star)}$ preserves $\mathcal{H}_{\text {disc }}$, providing us with a superselection rule. Again, this descends to a superselection rule for the physical operators on the physical Hilbert space.

However, this time the corresponding classical system has a similar feature $\left({ }^{2}\right)$. To see this, recall that when an operator $\widehat{A}$ is associated with a function $A$ on the classical phase space, the discrete eigenvalues of the operator $\widehat{A}$ are associated with parts of the phase space in which the orbits of the Hamiltonian vector field of the function $A$ are contained in compact regions, while the continuous eigenvalues are associated with parts of the phase space where these orbits are not contained in compact regions.

Suppose then that we have a single classical constraint $C$. For concreteness, we assume that the phase space $\Gamma$ is a finite dimensional manifold. Let $\Gamma_{\text {disc }}$ be the union of the collection of all orbits $O$ generated by this constraint such that there exists a compact $K_{O} \subset \Gamma$ containing $O$. We may think of $\Gamma_{\text {disc }}$ as the classical analogue of the space $\mathcal{H}_{\text {disc }}$ of discrete eigenvectors of $\widehat{C}$. Let $\Gamma_{\text {cont }}$ be the rest of the phase space $\Gamma$. Now, consider some function $A$ on the phase space such that $A$ Poisson commutes with $C$. The exponentiated action of the Hamiltonian vector field defined by $A$ is a (local) homeomorphism that maps orbits of $C$ onto orbits of $C$. Since, for an orbit $O \subset \Gamma_{\text {disc }}$, every neighborhood $U \subset \Gamma$ of $O$ contains some compact set $K_{U}$ which contains $O$, we therefore conclude that this exponentiated map cannot take an orbit $O_{\text {disc }} \subset \Gamma_{\text {disc }}$ to an orbit $O_{\text {cont }} \subset \Gamma_{\text {cont }}$ and vice versa. Thus, we find that (in the terminology of [13]) $\Gamma_{\text {disc }}$ and $\Gamma_{\text {cont }}$ contain disjoint sets of symplectic leaves of $\Gamma$ and we have a classical superselection law between the corresponding two parts of the reduced phase space. This seems to be the direct classical analogue of the quantum superselection rules discussed above; in fact, it is even stronger. All that is really required in the above argument is that the Poisson bracket of $A$ and $C$ vanish on the constraint surface. Thus, this superselection rule holds even for the so-called 'weak observables'.

It seems then that we must be careful. When the space $\Phi$ is chosen to reflect the smooth structure of the phase space, we have found physically meaningful superselection rules, a reasonable physical Hilbert space, and a (sufficiently) unique rigging map. However, when this is not the rationale for choosing $\Phi$, spurious results may occur. In the case of the diffeomorphism invariant states of [2], the corresponding $\Phi$ was chosen to reflect this structure as it is the appropriate domain of definition for the operators that were assumed to function as coordinates and momenta. Thus, within the framework of the auxiliary space of [2] and modulo questions concerning the Hamiltonian constraint (which was intentionally ignored), we expect that the superselection rules of [2] should

$\left(^{2}\right)$ The argument given below is an improved version of the one given in Appendix A of [2]. 
be taken seriously.

Acknowledgements. The author would like to thank Abhay Ashtekar, Petr Hajicek, Atsushi Higuchi, Nicholas Landsman, Jurek Lewandowski, Josè Mourão, and Thomas Thiemann for many useful discussions. Special thanks are due to Chris Isham and Karel Kuchař for repeatedly asking about the uniqueness of the physical Hilbert space given by the refined algebraic approach, to Chris Fewster for especially clarifying discussions and for help in locating reference [20], and to Carlo Rovelli for discussions on the significance of the superselection rules.

Appendix A. Uniqueness of the construction of Connection Representation Diffeomorphism Invariant States. In this appendix, we give a short proof that the rigging maps used in [2] to solve the diffeomorphism constraint completely exhaust the set of possible such maps given the choice of auxiliary space, the definitions of the quantum constraints, and the dense subspace $\Phi$ chosen in [2]. For a full definition of the terms and notation used below, see [2].

Recall from that the auxiliary Hilbert space of [2] is spanned by a set of orthonormal 'spin network states'. We shall denote these states by $\left|\Gamma_{\alpha, k}\right\rangle$, where $\alpha$ is a (piecewise analytic) graph embedded in a given analytic three manifold and $k$ is an index that takes some finite set of values (this set depends on the graph $\alpha$ ). In addition, (analytic) diffeomorphisms $\mathcal{D}$ act on these states by moving the graph $\alpha$ in the obvious way and permuting the values of the index $k$ allowed by $\alpha$.

The dense subspace $\Phi$ of Step $5 \mathrm{~b}$ is the space of so-called smooth cylindrical functions. This space contains all finite linear combinations of the spin network states $\Gamma_{\alpha, k}$ and, for our purposes, may in fact be identified with this slightly smaller space. Following [2], we shall consider only 'type $I$ graphs' (see [2]).

As in [2], it is convenient to introduce the subspaces $\mathcal{H}^{[\tilde{\beta}]}$ spanned by spin networks $\left|\Gamma_{\alpha, k}\right\rangle$ associated with graphs $\alpha$ that can be moved by a diffeomorphism to some graph $\beta$ for which $\tilde{\beta}$ is the 'maximal analytic extension'. These subspaces are superselected by the algebra $\mathcal{B}_{\text {phys }}^{(\star)}$ and, on each subspace, there is a corresponding map $\eta^{[\tilde{\beta}]}$ defined by:

$$
\eta^{[\tilde{\beta}]}|f\rangle=\left(\sum_{\mathcal{D}_{1} \in S(\tilde{\beta})} \sum_{\left[\mathcal{D}_{2}\right] \in G S(\tilde{\beta})} \mathcal{D}_{1} \mathcal{D}_{2}|f\rangle\right)^{\dagger}
$$

where we still need to introduce the set $S(\tilde{\beta})$ and the quotient space $G S(\tilde{\beta}) . S(\tilde{\beta})$ is chosen to be any set (and the above map does not depend on this choice) of diffeomorphisms $\mathcal{D}_{\tilde{\alpha}}$, one for each maximally extended analytic graph $\tilde{\alpha}$ diffeomorphic to $\tilde{\beta}$, such that $\mathcal{D}_{\tilde{\alpha}}$ moves the extended graph $\tilde{\beta}$ onto the extended graph $\tilde{\alpha}$. On the other hand, $G S(\tilde{\beta})$ (the 'graph symmetry group' of $\tilde{\beta})$ is the quotient $\operatorname{Iso}(\tilde{\beta}) / T A(\tilde{\beta})$ where the 'isotropy group' $\operatorname{Iso}(\tilde{\beta})$ is the group of diffeomorphisms which map $\tilde{\beta}$ onto $\tilde{\beta}$ and the 'trivial action group' $T A(\tilde{\beta})$ is the group of diffeomorphisms that map every edge $e$ in $\tilde{\beta}$ onto itself. In the formula above, $\left[\mathcal{D}_{1}\right]$ denotes the equivalence class of $\mathcal{D}_{2}$ in $G S(\tilde{\beta})$.

Any linear combination $\sum_{i \in I} a_{i} \eta^{\left[\tilde{\beta}_{i}\right]}$ with positive coefficients $a_{i}$ satisfies the requirements of Step 5c. (Note that this sum always converges no matter how big the coefficients 
$a_{i}$ or the index set $I$.) We would now like to show that such sums exhaust the set of all rigging maps. We will follow the same basic strategy as in the uniqueness proof of Section 3. That is, we now consider a generic map $\eta$ satisfying $5 \mathrm{c}$ and show that if $\eta^{[\tilde{\beta}]}|\phi\rangle=0$ for all $\tilde{\beta}$, then $\eta|\phi\rangle=0$ as well.

Suppose then that such that $\eta^{[\tilde{\beta}]}\left|\phi_{0}\right\rangle=0$ for all $\tilde{\beta}$. Since $\left|\phi_{0}\right\rangle \in \Phi$, it can be written as a sum of spin network states. It will be particularly convenient to write it in the form:

$$
\left|\phi_{0}\right\rangle=\sum_{i} \sum_{j} c_{i j} \mathcal{D}_{j}\left|\Gamma_{i}\right\rangle
$$

where $c_{i j} \in \mathbb{C}, \mathcal{D}_{j} \in \operatorname{Diff}^{\omega}$, and $\left\{\left|\Gamma_{i}\right\rangle\right\}$ is some set of spin network states, carefully chosen so that no analytic diffeomorphism maps one spin network state in this set onto another. Now, it is easily checked that $\left|\phi_{0}\right\rangle$ is annihilated by the above maps exactly when $\sum_{j} c_{i j}=0$ for each $i$. However, any rigging map that commutes with diffeomorphisms and whose image contains only diffeomorphism invariant states must also annihilate states with $\sum_{j} c_{i j}=0$. Thus, $\eta\left|\phi_{0}\right\rangle=0$.

Now consider some spin network state $\left|\Gamma_{0}\right\rangle:=\left|\Gamma_{\alpha, k}\right\rangle$ such that $\eta\left|\Gamma_{0}\right\rangle$ is nonzero (so that $\eta^{[\tilde{\alpha}]}\left|\Gamma_{0}\right\rangle$ is nonzero as well) and choose any other state $\left|\Gamma_{1}\right\rangle \in \mathcal{H}^{[\tilde{\alpha}]} \cap \Phi$. We want to construct an operator $A$ in $\mathcal{B}_{\text {phys }}^{(\star)}$ that has nonzero matrix elements between $\left|\Gamma_{0}\right\rangle$ and $\left|\Gamma_{1}\right\rangle$. This can be done by applying just the kind of 'group averaging' that was used in the construction of $\eta^{[\tilde{\beta}]}$ :

$$
A:=\sum_{\mathcal{D}_{1} \in S(\tilde{\alpha})} \sum_{\left[\mathcal{D}_{\in}\right] \in G S(\tilde{\alpha})} \mathcal{D}_{1} \mathcal{D}_{2}\left|\Gamma_{1}\right\rangle\left\langle\Gamma_{0}\right| \mathcal{D}_{2}^{-1} \mathcal{D}_{1}^{-1} .
$$

This operator is diffeomorphism invariant and finite on $\Phi$ for exactly the same reasons as the map $\eta^{[\tilde{\alpha}]}$ (and similarly for $A^{\dagger}$ ). As a result, it is an element of $\mathcal{B}_{\text {phys }}^{(\star)}$.

Note that $A\left|\Gamma_{0}\right\rangle$ is a sum of spin networks that differ from $\left|\Gamma_{1}\right\rangle$ only by a diffeomorphism. Thus, $A\left|\Gamma_{0}\right\rangle$ maps under $\eta$ to a diffeomorphism invariant state that is proportional to $\eta\left|\Gamma_{1}\right\rangle$. However, the number of terms in this sum is just the physical norm of the state $\left|\Gamma_{0}\right\rangle$ as defined through the map $\eta^{[\tilde{\alpha}]}$ (and similarly for $A^{\dagger}\left|\Gamma_{1}\right\rangle$ ). Let us therefore set $N_{0}=\left(\eta^{[\tilde{\alpha}]} \Gamma_{0}\right)\left[\Gamma_{0}\right]$ and $N_{1}=\left(\eta^{[\tilde{\alpha}]} \Gamma_{1}\right)\left[\Gamma_{1}\right]$ so that $\eta A^{\dagger} A\left|\Gamma_{0}\right\rangle=N_{0} \eta A^{\dagger}\left|\Gamma_{1}\right\rangle=N_{0} N_{1} \eta\left|\Gamma_{0}\right\rangle$. Applying this distribution to $\left|\Gamma_{0}\right\rangle$ we have:

$$
\frac{\left(\eta \Gamma_{1}\right)\left[\Gamma_{1}\right]}{N_{1}}=\frac{\left(\eta \Gamma_{0}\right)\left[\Gamma_{0}\right]}{N_{0}} .
$$

As before, this guarantees that when acting on the subspace $\mathcal{H}^{[\tilde{\alpha}]} \cap \Phi, \eta$ acts just like $\eta^{[\tilde{\alpha}]}$ up to an overall positive scale factor. Since the domains of the $\eta^{[\tilde{\alpha}]}$ 's are orthogonal, it follows that $\eta$ may in fact be expressed as a sum of the $\eta^{[\tilde{\alpha}]}$ weighted by positive coefficients.

Appendix B. Convergence of the group averaging procedure. In this appendix we show that the integral that defines the group averaged inner product does not (absolutely) converge on the entire space $\Phi$ given in the second example of Section 4. Recall that the group averaging proposal $[2,8,9,12]$ is to introduce the physical inner product

$$
\langle\phi, \psi\rangle_{\mathrm{phys}}=\int d t\left\langle\phi, e^{i t \widehat{C}} \psi\right\rangle_{\mathrm{phys}}
$$


for $\phi, \psi$ in $\Phi$. If this integrand is in fact $L^{1}$, then we may write this as

$$
\lim _{T \rightarrow \infty} \int_{-T}^{T}\left\langle\phi, e^{i t \widehat{C}} \psi\right\rangle=\lim _{T \rightarrow \infty}\left\langle\phi, \frac{\sin (T \widehat{C})}{\widehat{C}} \psi\right\rangle=\lim _{T \rightarrow \infty} \int_{\Lambda} d \lambda\left\langle\phi(\lambda), \frac{\sin (T \lambda)}{\lambda} \psi(\lambda)\right\rangle_{\lambda} .
$$

However, we will now show that this limit fails to exist for general $\phi, \psi \in \Phi$.

For convenience, we assume that $D_{C}=\mathbb{R}$. Furthermore, we will take $a=1$ and introduce the intervals $J_{n}^{-}=\left(-2^{n+1},-2^{n}\right), J_{n}^{+}=\left(2^{n}, 2^{n+1}\right)$. Finally, let $R_{E}^{\prime}=R_{E} \cup$ $\left(\bigcup_{ \pm, \text {even } n} J_{n}^{ \pm}\right), R_{O}^{\prime}=R_{O} \cup\left(\bigcup_{ \pm, \text {odd } n} J_{n}^{ \pm}\right)$, and let $|\psi\rangle \in \Phi$ be any state such that $\psi(c, k)$ vanishes for $c$ in $R_{O}^{\prime}$.

The important property of $R_{E}^{\prime}$ is that this set is preserved when the real line is scaled by a factor of $2^{k}$. As such, given any function $\psi(\lambda)$ which is continuous on $R_{E}^{\prime}$, the limit

$$
\lim _{k \rightarrow \infty} \int_{R_{E}^{\prime}} \frac{\psi(\lambda) \sin \left(2^{k} T \lambda\right)}{\lambda}
$$

for large $k$ is just

$$
\psi(0) \int_{R_{E}^{\prime}} \frac{\sin \left(2^{k} T \lambda\right)}{\lambda} \equiv \psi(0) I(T)
$$

which is independent of $k$. It follows that the limit exists for large $T$ if and only if $I(T)$ is constant.

However, we will now show that $I(T)$ is not constant. Note that its derivative is

$$
d I / d T=\int_{R_{E}^{\prime}} \cos (T \lambda) d \lambda
$$

and suppose that $T=\pi$. Then, $\int_{J_{n}^{ \pm}} \cos (\pi \lambda) d \lambda=0$, but we have $\int_{I_{n}^{ \pm}} \cos (\pi \lambda)>0$ so that $I(\pi)>0$. As a result, the group averaging norm does not (absolutely) converge for any nontrivial $|\psi\rangle \in \Phi$ that vanishes on $R_{O}^{\prime}$.

\section{References}

[1] A. Ashtekar, Non-Perturbative Canonical Gravity, Lectures notes prepared in collaboration with R. S. Tate, World Scientific, Singapore, 1991.

[2] A. Ashtekar, J. Lewandowski, D. Marolf, J. Mourão, and T. Thiemann, Quantization of diffeomorphism invariant theories of connections with local degrees of freedom, J. Math. Phys. 36 (1995), 6456-6493; gr-qc/9504018.

[3] A. Ashtekar and R. S. Tate, An algebraic extension of Dirac quantization: Examples, J. Math. Phys. 35 (1994), 6434-6470.

[4] B. DeWitt, Quantum Theory of Gravity. I: The Canonical Theory, Phys. Rev. (2) 160 (1967), 1113-1148.

[5] P. A. M. Dirac, Lectures on Quantum Mechanics, Belfer Graduate School of Science, Yeshiva University, New York, 1964.

[6] I. M. Gel'fand, N. Ya. Vilenkin, Generalized Functions: vol. 4, Applications of Harmonic Analysis, Academic Press, New York, London, 1964.

[7] P. Hájíček, Quantization of Systems with Constraints, in: Canonical Gravity: from classical to quantum, J. Ehlers, H. Friedrich (eds.), Lecture Notes in Phys. 434, Springer, Berlin, 1994, 113-149. 
[8] A. Higuchi, Quantum linearization instabilities of de Sitter spacetime: II, Classical Quantum Gravity 8 (1991), 1983-2004.

[9] A. Higuchi, Linearized quantum gravity in flat space with toroidal topology, Classical Quantum Gravity 8 (1991), 2023-2034.

[10] C. Isham, Canonical Gravity and the Problem of Time, Imperial College, preprint TP/9192/25; gr-qc/9210011, 1992.

[11] K. Kuchař, Time and Interpretations of Quantum Gravity, in: Proceedings of the 4th Canadian Conference on General Relativity and Relativistic Astrophysics, G. Kunstatter et al. (eds.), World Scientific, New Jersey, 1992, 211-314.

[12] N. Landsman, Rieffel induction as generalized quantum Marsden-Weinstein reduction, J. Geom. Phys. 15 (1995), 285-319; hep-th/9305088. Erratum: ibid. 17 (1995), 298.

[13] N. Landsman, Classical and quantum representation theory, in: Proceedings Seminar Mathematical Structures in Field Theory, E. A. de Kerf and H. G. J. Pijls (eds.), CWIsyllabus, CWI, Amsterdam, to appear.

[14] N. Landsman and U. Wiedemann, Massless Particles, Electromagnetism, and Rieffel Induction, Rev. Math. Phys. 7 (1995), 923-958; hep-th/9411174.

[15] D. Marolf, The spectral analysis inner product for quantum gravity, preprint gr-qc/9409036, to appear in the Proceedings of the VIIth Marcel-Grossman Conference, R. Ruffini and M. Keiser (eds.), World Scientific, Singapore, 1995.

[16] D. Marolf, Green's Bracket Algebras and their Quantization, Ph. D. Dissertation, The University of Texas at Austin, 1992.

[17] D. Marolf, Quantum observables and recollapsing dynamics, Classical Quantum Gravity 12 (1995), 1199-1220; gr-qc/9404053.

[18] D. Marolf, Observables and a Hilbert Space for Bianchi IX, Classical Quantum Gravity 12 (1995), 1441-1454; gr-qc/9409049.

[19] D. Marolf, Almost Ideal Clocks in Quantum Cosmology: A Brief Derivation of Time, Classical Quantum Gravity 12 (1995), 2469-2486; gr-qc/9412016.

[20] B. Simon, Quantum Mechanics for Hamiltonians defined as quadratic forms, Princeton Univ. Press, Princeton, 1971, p. 120. 